\title{
The backreaction of anti-M2 branes on a warped Stenzel space
}

\author{
losif Bena, ${ }^{a}$ Gregory Giecold ${ }^{a}$ and Nick Halmagyi ${ }^{a, b}$ \\ ${ }^{a}$ Institut de Physique Théorique, CEA Saclay, CNRS URA 2306, \\ F-91191 Gif-sur-Yvette, France \\ ${ }^{b}$ Laboratoire de Physique Théorique et Hautes Energies, \\ Université Pierre et Marie Curie, CNRS UMR 7589, \\ F-75252 Paris Cedex 05, France \\ E-mail: iosif.bena@cea.fr, gregory.giecold@cea.fr, \\ halmagyi@lpthe.jussieu.fr
}

ABSTRACT: We find the superpotential governing the supersymmetric warped M-theory solution with a transverse Stenzel space found by Cvetič, Gibbons, Lü and Pope in hepth/0012011, and use this superpotential to extract and solve the twelve coupled equations underlying the first-order backreacted solution of a stack of anti-M2 branes in this space. These anti-M2 branes were analyzed recently in a probe approximation by Klebanov and Pufu, who conjectured that they should be dual to a metastable vacuum of a supersymmetric $2+1$ dimensional theory. We find that the would-be supergravity dual to such a metastable vacuum must have an infrared singularity and discuss whether this singularity is acceptable or not. Given that a similar singularity appears when placing anti-D3 branes in the Klebanov-Strassler solution, our work strengthens the possibility that anti-branes in warped throats do not give rise to metastable vacua.

KEYWords: Gauge-gravity correspondence, Supersymmetry Breaking, dS vacua in string theory, AdS-CFT Correspondence

ARXIV EPRINT: 1011.2195 


\section{Contents}

1 Introduction and discussion $\quad 1$

2 Perturbations around a supersymmetric solution $\quad 5$

2.1 The first-order Borokhov-Gubser formalism 6

$\begin{array}{lll}2.2 & \text { The perturbation ansatz } & 7\end{array}$

2.3 The supersymmetric background 8

2.4 Explicit equations 9

$2.4 .1 \xi_{a}$ equations 9

$\begin{array}{lll}2.4 .2 & \phi^{a} \text { equations } & 9\end{array}$

3 The force on a probe M2 10

4 The space of solutions $\quad 11$

$\begin{array}{lll}4.1 & \text { Analytic solutions for the } \tilde{\xi} \text { 's } & 12\end{array}$

$\begin{array}{ll}4.2 & \text { Solving the } \phi^{i} \text { equations } \\ 4.2 .13\end{array}$

$\begin{array}{lll}\text { 4.2.1 The space of solutions } & 13\end{array}$

$\begin{array}{lll}4.2 .2 & \text { IR behavior } & 14\end{array}$

$\begin{array}{lll}\text { 4.2.3 UV behavior } & 15\end{array}$

5 Boundary conditions for M2 branes $\quad 18$

$\begin{array}{lll}5.1 & \text { BPS M2 branes } & 18\end{array}$

6 Constructing the anti-M2 brane solution 20

$\begin{array}{lll}\text { A } & \text { Subtleties in section } 2 & 22\end{array}$

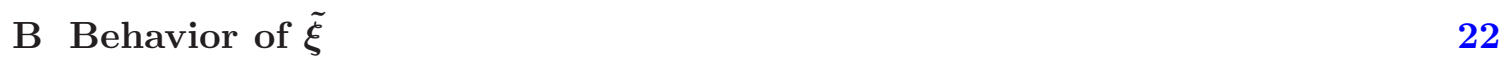

B.1 IR behavior of $\tilde{\xi} \quad 23$

B.2 UV behavior of $\tilde{\xi} \quad 23$

\section{Introduction and discussion}

The recent revival of interest in metastable supersymmetry breaking in quantum field theory is largely due to the work of Intriligator, Seiberg and Shih [1] (ISS). This work presents a mechanism to naturally circumvent some of the problems afflicting other models for dynamic supersymmetry breaking (DSB) [2-5]. A natural question that was posed immediately after [1] is whether metastable vacua also exist in string realizations of supersymmetric field theories. 
For type IIA brane-engineering models of supersymmetric field theories, the answer to this question is negative [6]. Indeed, these models are constructed using D4 branes ending on codimension-two defects inside NS5 branes [6-8], which source NS5 worldvolume fields that grow logarithmically at infinity. In supersymmetric vacua this logarithmic growth encodes the running of the gauge theory coupling constant with the energy [9-12], but these logarithmic modes are different in the candidate metastable brane configuration and in the supersymmetric one. This implies that the candidate metastable brane configuration and the supersymmetric one differ by an infinite amount, and hence cannot decay into each other. Hence, the type IIA brane construction does not describe a metastable vacuum of a supersymmetric theory, but instead a nonsupersymmetric vacuum of a nonsupersymmetric theory.

Another arena where one might try to find string theory realizations of metastable vacua are IIB holographic duals of certain supersymmetric gauge theories. The best-known example in this class was proposed by Kachru, Pearson and Verlinde [13, 14], who argued that a background with anti-D3 branes at the bottom of the Klebanov-Strassler warped deformed conifold [15] is dual to a metastable vacuum of the dual supersymmetric gauge theory. Since the Klebanov-Strassler solution has positive D3 brane charge dissolved in flux, the anti-D3 branes can annihilate against this charge (this annihilation happens via the polarization of the anti-D3 branes into an NS5 brane $[16,17]$ ), and this bulk process is argued to correspond to the decay of the metastable vacuum to the supersymmetric one in the dual field theory.

Another proposal for a metastable vacuum obtained by putting anti-branes at the bottom of a smooth warped throat with positive brane charge dissolved in flux has recently been made by Klebanov and Pufu [18], who argued that probe anti-M2 branes at the tip of a supersymmetric warped M-theory background with transverse Stenzel space [19], give rise to a long-lived metastable vacuum. The supersymmetric solution, first found by Cvetič, Gibbons, Lü and Pope (CGLP) in [20] has M2 charge dissolved in fluxes and a large $S^{4}$ in the infrared. The anti-branes can annihilate against the charge dissolved in fluxes by polarizing into M5 branes [21] wrapping three-spheres inside the $S^{4}$.

The probe brane analyses described above, while indicative that a metastable vacuum might exist, are however not enough to establish this. One possible issue which can cause the backreacted solution to differ significantly from the probe analysis is the presence of non-normalizable modes. If the anti-branes indeed source such modes then the candidate metastable configuration is not dual to a non-supersymmetric vacuum of a supersymmetric theory, but to a non-supersymmetric vacuum of a non-supersymmetric theory, and the supersymmetry breaking is not dynamical but explicit. The existence of non-normalizable modes is not visible in the probe approximation (much like the existence of type IIA log-growing modes was not visible in $g_{s}=0$ brane constructions $[7,8]$ ), but only upon calculating the backreaction of the probe branes - a not too easy task.

In [22] two of the authors and M. Graña found the possible first-order backreacted solution sourced by a stack of anti-D3 branes smeared on the large $S^{3}$ at the bottom of the Klebanov-Strassler (KS) solution, and found two very interesting features: first, of the 14 physical modes describing $\mathrm{SU}(2) \times \mathrm{SU}(2) \times Z_{2}$-invariant perturbations of the warped 
deformed conifold, only one mode enters in the expression of the force that a probe D3 brane feels in this background. Hence, since anti-D3 branes attract probe branes, if the perturbed solution is to have any chance to describe backreacted anti-D3 branes, this mode must be present. ${ }^{1}$ The second feature of this solution is that if the force mode is present, the infrared ${ }^{2}$ must contain a certain singularity, which has finite action. ${ }^{3}$ Note that having a finite action does not automatically make a singularity acceptable - negative-mass Schwarzschild is an obvious counterexample [27]. As discussed in [22], if this singularity is unphysical, then the solution sourced by the anti-D3 branes cannot be thought of as a small perturbation of the KS solution, and therefore does not describe a metastable vacuum of the dual theory. If this singularity is physical, the first-order solution does describe anti-D3 branes at the bottom of the KS solution, and work is in progress to determine what are the features of this solution, and whether the perturbative anti-D3 brane solution describes or not metastable vacua of the dual theory.

The purpose of this paper is to calculate the first-order backreaction of the other proposed metastable configuration with anti-branes in a background with charge dissolved in fluxes: the anti-M2 branes in the Stenzel-CGLP solution [20]. In order to do this we smear the anti-M2 branes on the large $S^{4}$ at the bottom of the Stenzel-CGLP solution, and solve for all possible deformations of this background that preserve its $\mathrm{SO}(5)$ symmetry. We consider an ansatz for these deformations; the space of deformations is parameterized by 6 functions of one variable satisfying second-order differential equations. However, when perturbing around a supersymmetric solution, Borokhov and Gubser [28] have observed that these second-order equations factorize into first-order ones, that are much easier to solve. Nevertheless, in order to apply the Borokhov-Gubser method, one needs to find the superpotential underlying the supersymmetric solution, which for the warped fluxed Stenzel-CGLP solution was not known until now. The first result of this paper, presented in section 2 , is to find this superpotential, ${ }^{4}$ and derive two sets of first-order equations governing the space of deformations.

We then show in section 3 that the force felt by a probe M2 brane in the most general perturbed background depends on only one of the "conjugate-momentum" functions that appear when solving the first-order system, and hence on only one of the 10 constants parameterizing the deformations around the supersymmetric solution. We then solve in section 4 the two sets of first-order differential equations. Amazingly enough, the solutions for the first set of equations (for the conjugate-momentum functions) can be found explicitly in terms of incomplete elliptic integrals (a huge improvement on the situation in [22]). We also find the homogeneous solutions to the other equations and give implicitly the full solution to the system in terms of integrals. We also provide the explicit UV and IR

\footnotetext{
${ }^{1}$ The asymptotic behavior of the force matches the one argued for in [23], and the existence of this mode was first intuited in [24] which set out to study the UV asymptotics of the perturbations corresponding to anti-D3 branes in the KT background [25].

${ }^{2} \mathrm{An}$ IR analysis of some of the non-supersymmetric isometry-preserving perturbations of the KlebanovStrassler background can also be found in [26].

${ }^{3}$ This was first observed by I. Klebanov.

${ }^{4}$ This is the equivalent of the Papadopoulos-Tseytlin superpotential for the KS solution [29-31].
} 
expansions of the full space of deformations, and find which deformations correspond to normalizable modes and which deformations correspond to non-normalizable modes.

In section 5 we then use the machinery we developed to recover the perturbative expansion of the known solution sourced by BPS M2 branes smeared on the $S^{4}$ at the tip of the Stenzel-CGLP solution [20], and analyze the infrared of the possible solution sourced by anti-M2 branes. After removing some obviously unphysical divergences and demanding that in the first-order backreacted solution a probe M2 brane feels a nonzero force, we find that the only backreacted solution that can correspond to anti-M2 branes must have an infrared singularity, coming from a four-form field strength with two or three legs on the three-sphere that is shrinking to zero size at the tip of the Stenzel space.

Hence, the first-order backreacted solution for the anti-M2 branes has the same two key features as the anti-D3 branes in KS: the force felt by a probe M2 brane in this background depends only on one of the 10 physical perturbation modes around this solution, and the solution where the force-carrying mode is turned on must have an infrared singularity coming from a divergent energy in the M-theory four-form field strength. Nevertheless, unlike in the "anti-D3 in KS" solution, the action of this infrared singularity also diverges. Again, if this singularity is physical, our first-order backreacted solution describes anti-M2 branes in the CGLP background, and, to our knowledge, would be the first backreacted supergravity solution dual to metastable susy-breaking in $2+1$ dimensions since the work of Maldacena and Năstase [32]. This may be of interest both in the programme of using the AdS/CFT correspondence to describe strongly-interacting condensed-matter systems, and also in view of the relevance of three-dimensional QFT's at strong coupling to a recent holographic model of four-dimensional cosmology [33]. On the other hand, if the singularity is not physical then the backreaction of the anti-M2 branes cannot be taken into account perturbatively; this indicates that the only solution with proper anti-M2 brane boundary conditions in the infrared is the solution for anti-M2 branes in a CGLP background with anti-M2 brane charge dissolved in flux, and hence the anti-M2 branes flip the sign of the M2 brane charge dissolved in flux.

Given the similarity of the results of the "anti-D3 in KS" and of the "anti-M2 in CGLP" analyses and the drastically-different calculations leading to them, it is rather natural to expect that the underlying physics of the two setups is the same: either both singularities are physical, which indicates that anti-branes in backgrounds with charge dissolved in fluxes give rise to metastable vacua, or they are both unphysical, which supports the idea that anti-branes in such backgrounds cannot be treated as a perturbation of the original solution, and may flip the sign of the charge dissolved in flux. Furthermore, our analysis suggests that one cannot use the finiteness of the action as a criterion for accepting a singularity. This would allow the anti-D3 singularity and exclude the anti-M2 one, which would be rather peculiar, given the striking resemblance of the two systems.

There are a few possible explanations for the singularities we encounter in the antiM2 and anti-D3 solutions. One is that these singularities are accompanied by stronger, physical singularities, coming from the smeared anti-M2 or anti-D3 sources, and one can hope that whatever mechanism renders the stronger singularities physical may cure the subleading ones as well. Another explanation is that the subleading singularities are a 
result of smearing the antibranes. This is a difficult argument to support with calculational evidence, as the unsmeared solution is a formidable problem even for BPS branes in Stenzel spaces [34, 35]. Furthermore, a naive comparison of the anti-M2 and anti-D3 solutions indicates that the stronger the physical singularity associated with the brane sources is, the stronger the subleading singularity will be. Hence, it is likely that unsmearing will make things worse, not better, as is illustrated for a class of non-BPS $\mathrm{AdS}_{4}$ solutions in reference [36]. Note also that one cannot link the divergent four-form field strength with the M5 branes into which the anti-M2 branes at the tip of the Stenzel-CGLP solution polarize - they have incompatible orientations.

It is also interesting to remember that when one attempts to build string realisations of four-dimensional metastable vacua, either via brane constructions [6] or via AdS-CFT [22], the non-normalizable modes one encounters are log-growing modes, which one could in hindsight have expected from the generic running of coupling constants of four-dimensional gauge theories with the energy.

For anti-M2 branes there is no such link. There exist both AdS/CFT duals of metastable vacua of $2+1$ dimensional gauge theories [32], as well as brane-engineering constructions of such metastable vacua (using D3 branes ending on codimension-three defects inside NS5 branes) [37]. The nonexistence of an anti-M2 metastable vacuum could only be seen in supergravity, and comes from the way the fields of the anti-M2 brane interact with the magnetic fields that give rise to the charge dissolved in fluxes. This may indicate there is a problem with trying to construct metastable vacua in string theory by putting antibranes in backgrounds with charge dissolved in fluxes. In an upcoming paper [38] we will also argue that anti-D2 branes in backgrounds with D2 brane charge dissolved in fluxes [39], that one of us investigated in [40], have similar problems.

\section{Perturbations around a supersymmetric solution}

We are interested in the backreaction of a set of anti-M2 branes spread on a four-sphere at the bottom of the warped Stenzel geometry [19] with nontrivial fluxes. Smearing the anti-M2's is necessary in order for the perturbed solution to have the same $\mathrm{SO}(5)$ global symmetry as the supersymmetric solution of Cvetič, Gibbons, Lü and Pope (CGLP) [20]. The perturbed metric and flux coefficients are then functions of only one radial variable, and generically satisfy $n$ second-order differential equations.

However, when perturbing around a supersymmetric solution governed by a superpotential, Borokhov and Gubser [28] have observed that these $n$ second-order equations factorize into $n$ first-order equations for certain momenta and $n$ first-order equations for the metric and flux coefficients, and that furthermore the $n$ equations for the momenta do not contain the metric and flux coefficients, and hence can be solved independently. This technique has been used in several related works $[22,28,41]$ and we consider this to be the technique of choice for deformation problems that depend on just one coordinate. 


\subsection{The first-order Borokhov-Gubser formalism}

While the following summary can be found by now in several sources, we include it here for completeness. When the equations of motion governing the fields $\phi^{a}$ of a certain supersymmetric solution come from the reduction to a one-dimensional Lagrangian

$$
\mathcal{L}=-\frac{1}{2} G_{a b} \frac{d \phi^{a}}{d \tau} \frac{d \phi^{b}}{d \tau}-V(\phi)
$$

whose potential $V(\phi)$ comes from a superpotential,

$$
V(\phi)=\frac{1}{8} G^{a b} \frac{\partial W}{\partial \phi^{a}} \frac{\partial W}{\partial \phi^{b}}, .
$$

The Lagrangian is written as

$$
\mathcal{L}=-\frac{1}{2} G_{a b}\left(\frac{d \phi^{a}}{d \tau}-\frac{1}{2} G^{a c} \frac{\partial W}{\partial \phi^{c}}\right)\left(\frac{d \phi^{a}}{d \tau}-\frac{1}{2} G^{a c} \frac{\partial W}{\partial \phi^{c}}\right)-\frac{d W}{d \tau},
$$

and the supersymmetric solutions satisfy

$$
\frac{d \phi^{a}}{d \tau}-\frac{1}{2} G^{a b} \frac{\partial W}{\partial \phi^{b}}=0 .
$$

We now want to find a perturbation in the fields $\phi^{a}$ around their supersymmetric background value $\phi_{0}^{a}$

$$
\phi^{a}=\phi_{0}^{a}+\phi_{1}^{a}(X)+\mathcal{O}\left(X^{2}\right),
$$

where $X$ represents the set of perturbation parameters in which $\phi_{1}^{a}$ is linear. The deviation from the gradient flow equations for the perturbation $\phi_{1}^{a}$ is measured by the conjugate momenta $\xi_{a}$

$$
\begin{aligned}
\xi_{a} & \equiv G_{a b}\left(\phi_{0}\right)\left(\frac{d \phi_{1}^{b}}{d \tau}-M_{d}^{b}\left(\phi_{0}\right) \phi_{1}^{d}\right), \\
M_{d}^{b} & \equiv \frac{1}{2} \frac{\partial}{\partial \phi^{d}}\left(G^{b c} \frac{\partial W}{\partial \phi^{c}}\right) .
\end{aligned}
$$

The $\xi_{a}$ are linear in the expansion parameters $X$, hence they are of the same order as the $\phi_{1}^{a}$. When all the $\xi_{a}$ vanish the deformation is supersymmetric.

The main point of this construction is that the second-order equations of motion governing the perturbations reduce to a set of first-order linear equations for $\left(\xi_{a}, \phi^{a}\right)$ :

$$
\begin{aligned}
\frac{d \xi_{a}}{d \tau}+\xi_{b} M^{b}{ }_{a}\left(\phi_{0}\right) & =0, \\
\frac{d \phi_{1}^{a}}{d \tau}-M_{b}^{a}\left(\phi_{0}\right) \phi_{1}^{b} & =G^{a b} \xi_{b} .
\end{aligned}
$$

Note that equation (2.9) is just a rephrasing of the definition of the $\xi_{a}$ in (2.6), while (2.8) implies the equations of motion. Since one considers these perturbations in a metric ansatz in which the reparametrization invariance of the radial variable is fixed, in addition to these equations one must enforce the zero-energy condition

$$
\xi_{a} \frac{d \phi_{0}^{a}}{d r}=0
$$




\subsection{The perturbation ansatz}

Using the analysis of the CGLP solution in [18], one can easily see that the ansatz for the $\mathrm{SO}(5)$-invariant eleven-dimensional supergravity solution we are looking for is

$$
\begin{aligned}
d s^{2} & =e^{-2 z(r)} d x_{\mu} d x^{\mu}+e^{z(r)}\left[e^{2 \gamma(r)} d r^{2}+e^{2 \alpha(r)} \sigma_{i}^{2}+e^{2 \beta(r)} \tilde{\sigma}_{i}^{2}+e^{2 \gamma(r)} \nu^{2}\right] \\
G_{4} & =d K(\tau) \wedge d x^{0} \wedge d x^{1} \wedge d x^{2}+m F_{4},
\end{aligned}
$$

where $F_{4}=d A_{3}$ and

$$
\begin{aligned}
A_{3}= & f(r) \tilde{\sigma}_{1} \wedge \tilde{\sigma}_{2} \wedge \tilde{\sigma}_{3}+h(r) \epsilon^{i j k} \sigma_{i} \wedge \sigma_{j} \wedge \tilde{\sigma}_{k} \\
\Rightarrow F_{4}= & f^{\prime} d r \wedge \tilde{\sigma}_{1} \wedge \tilde{\sigma}_{2} \wedge \tilde{\sigma}_{3}+h^{\prime} \epsilon^{i j k} d r \wedge \sigma_{i} \wedge \sigma_{j} \wedge \tilde{\sigma}_{k} \\
& +\frac{1}{2}(4 h-f) \epsilon^{i j k} \nu \wedge \sigma_{i} \wedge \tilde{\sigma}_{j} \wedge \tilde{\sigma}_{k}-6 h \nu \wedge \sigma_{1} \wedge \sigma_{2} \wedge \sigma_{3} .
\end{aligned}
$$

Our notation for the one-forms on the Stenzel space is by now standard [18], in the sense that with the definitions

$$
\sigma_{i}=L_{1 i}, \quad \tilde{\sigma}_{i}=L_{2 i}, \quad \nu=L_{12},
$$

they satisfy

$$
\begin{aligned}
d \sigma_{i} & =\nu \wedge \tilde{\sigma}_{i}+L_{i j} \wedge \sigma_{j}, \\
d \tilde{\sigma}_{i} & =-\nu \wedge \sigma_{i}+L_{i j} \wedge \tilde{\sigma}_{j}, \\
d \nu & =-\sigma_{i} \wedge \tilde{\sigma}_{i}, \\
d L_{i j} & =L_{i k} \wedge L_{k j}-\sigma_{i} \wedge \sigma_{j}-\tilde{\sigma}_{i} \wedge \tilde{\sigma}_{j} .
\end{aligned}
$$

Integrating one particular component of the equation of motion for the flux

$$
d * G_{4}=\frac{1}{2} G_{4} \wedge G_{4}
$$

gives

$$
K^{\prime}=6 m^{2}\left[h(f-2 h)-\frac{1}{54}\right] e^{-3(\alpha+\beta)-6 z},
$$

where we have chosen the integration constant such that the BPS solution [20] is regular, i.e. there are no explicit source M2 branes. We refer to appendix A for more details.

Performing a standard dimensional reduction on this ansatz down to one dimension, we obtain the following Lagrangian

$$
L=\left(T_{g r}+T_{\text {mat }}\right)-\left(V_{g r}+V_{\text {mat }}\right)
$$

with the gravitational and matter sectors given by

$$
\begin{aligned}
& T_{g r}=3 e^{3(\alpha+\beta)}\left[\alpha^{\prime 2}+\beta^{\prime 2}-\frac{3}{4} z^{\prime 2}+3 \alpha^{\prime} \beta^{\prime}+\alpha^{\prime} \gamma^{\prime}+\beta^{\prime} \gamma^{\prime}\right], \\
& V_{g r}=\frac{3}{4} e^{\alpha+\beta}\left[e^{4 \alpha}+e^{4 \beta}+e^{4 \gamma}-2 e^{2 \alpha+2 \beta}-6 e^{2 \alpha+2 \gamma}-6 e^{2 \beta+2 \gamma}\right]
\end{aligned}
$$


and

$$
\begin{aligned}
T_{\text {mat }}= & -\frac{m^{2}}{4} e^{3 \alpha+\beta-3 z}\left(f^{\prime 2} e^{-4 \beta}+12 h^{\prime 2} e^{-4 \alpha}\right), \\
V_{\text {mat }}= & 3 m^{2} e^{\alpha+3 \beta-3 z}\left[3 h^{2} e^{-4 \alpha}+\frac{1}{4}(4 h-f)^{2} e^{-4 \beta}\right] \\
& +9 m^{4} e^{-3(\alpha+\beta+2 z)}\left[h(f-2 h)-\frac{1}{54}\right]^{2} .
\end{aligned}
$$

The superpotential is given by

$$
W=-3 e^{2 \alpha+2 \beta}\left(e^{2 \alpha}+e^{2 \beta}+e^{2 \gamma}\right)-6 m^{2} e^{-3 z}\left[h(f-2 h)-\frac{1}{54}\right] .
$$

It is worth noting that equation (2.2) only defines the superpotential up one independent minus sign which can then be absorbed in (2.8) and (2.9) by changing the sign of the radial variable and the $\xi_{a}$. However, with the wisdom of hindsight, we choose a radial variable such that fields decay at infinity and not minus infinity, thus simultaneously fixing the sign of the superpotential.

\subsection{The supersymmetric background}

Here we summarize the expressions that the fields in our ansatz take when specialized to the zeroth-order CGLP solution [20] around which we endeavor to study supersymmetric and non-supersymmetric perturbations.

We should note that the CGLP solution with transverse Stenzel geometry is to the warped M-theory solution with transverse Stiefel space [42] what the IIB Klebanov-Strassler solution [15] and the deformed conifold [43] are to the Klebanov-Tseytlin solution [25] and the singular conifold. The Stenzel space is a higher-dimensional generalization of the deformed conifold. A useful summary of many details of the supergravity solution can be found in [44] and proposals for the dual field theory can be found in [44, 45]

The supersymmetric solution around which we will perturb was found in [20]. It can be summarized in our ansatz by

$$
\begin{aligned}
e^{2 \alpha_{0}} & =\frac{1}{3}(2+\cosh (2 r))^{1 / 4} \cosh (r), \\
e^{2 \beta_{0}} & =\frac{1}{3}(2+\cosh (2 r))^{1 / 4} \sinh (r) \tanh (r), \\
e^{2 \gamma_{0}} & =(2+\cosh (2 r))^{-3 / 4} \cosh ^{3}(r), \\
f_{0} & =\frac{1}{3^{3 / 2}} \frac{\left(1-3 \cosh ^{2}(r)\right)}{\cosh ^{3}(r)} \\
h_{0} & =-\frac{1}{3^{3 / 2}} \frac{1}{\cosh (r)}, \\
e^{3 z_{0}(y)} & =2^{5 / 2} 3 m^{2} \int_{y}^{\infty} \frac{d u}{\left(u^{4}-1\right)^{5 / 2}},
\end{aligned}
$$

where

$$
y^{4} \equiv 2+\cosh (2 r)
$$


With this change of coordinate we can write

$$
e^{3 z_{0}}=\sqrt{2} m^{2} \frac{y\left(7-5 y^{4}\right)}{\left(y^{4}-1\right)^{3 / 2}}+5 \sqrt{2} m^{2} F\left(\arcsin \left(\frac{1}{y}\right) \mid-1\right),
$$

where the incomplete elliptic integral of the first kind is

$$
F(\phi \mid q)=\int_{0}^{\phi}\left(1-q \sin (\theta)^{2}\right)^{-1 / 2} d \theta
$$

and we have fixed the integration constant (denoted $c_{0}$ in [20]) by requiring $e^{3 z_{0}} \rightarrow 0$ as $r \rightarrow \infty$.

\subsection{Explicit equations}

We now write out explicitly the two sets of equations (2.8) and (2.9). In both cases a particular field redefinition simplifies things substantially.

\subsection{1 $\xi_{a}$ equations}

The $\xi^{a}$ equations (2.8) simplify in the basis

$$
\tilde{\xi}_{a}=\left(\xi_{1}+\xi_{2}+\xi_{3}, \xi_{1}-\xi_{2}+3 \xi_{3}, \xi_{1}+\xi_{2}-3 \xi_{3}, \xi_{4}, \xi_{5}, \xi_{6}\right)
$$

In the order which we solve them, the equations are

$$
\begin{aligned}
& \tilde{\xi}_{4}^{\prime}=6 m^{2} e^{-3\left(\alpha_{0}+\beta_{0}+z_{0}\right)}\left(\left(f_{0}-2 h_{0}\right) h_{0}-\frac{1}{54}\right) \tilde{\xi}_{4} \\
& \tilde{\xi}_{1}^{\prime}=12 m^{2} e^{-3\left(\alpha_{0}+\beta_{0}+z_{0}\right)}\left(\left(f_{0}-2 h_{0}\right) h_{0}-\frac{1}{54}\right) \tilde{\xi}_{4} \\
& \tilde{\xi}_{5}^{\prime}=\frac{1}{2} e^{\alpha_{0}-\beta_{0}} \tilde{\xi}_{6}-2 m^{2} h_{0} e^{-3\left(\alpha_{0}+\beta_{0}+z_{0}\right)} \tilde{\xi}_{4} \\
& \tilde{\xi}_{6}^{\prime}=6 e^{-3\left(\alpha_{0}-\beta_{0}\right)} \tilde{\xi}_{5}-2 e^{\alpha_{0}-\beta_{0}} \tilde{\xi}_{6}-2 m^{2} e^{-3\left(\alpha_{0}+\beta_{0}+z_{0}\right)}\left(f_{0}-4 h_{0}\right) \tilde{\xi}_{4}, \\
& \tilde{\xi}_{3}^{\prime}=\frac{2}{9} e^{-3\left(\alpha_{0}+\beta_{0}+z_{0}\right)}\left[18 e^{2\left(\alpha_{0}+\beta_{0}+\gamma_{0}\right)+3 z_{0}} \tilde{\xi}_{3}+m^{2}\left(54 h_{0}\left(f_{0}-2 h_{0}\right)-1\right) \tilde{\xi}_{4}\right], \\
& \tilde{\xi}_{2}^{\prime}=\frac{1}{2} e^{-3 \alpha_{0}-\beta_{0}}\left[2 e^{2\left(\alpha_{0}+\beta_{0}\right)} \tilde{\xi}_{2}-6 e^{2\left(\alpha_{0}+\gamma_{0}\right)} \tilde{\xi}_{3}-72 h_{0} e^{4 \beta_{0}} \tilde{\xi}_{5}\right. \\
& \left.\quad+e^{4 \alpha_{0}}\left(-3 \tilde{\xi}_{1}+2 \tilde{\xi}_{2}+3 \tilde{\xi}_{3}+2\left(f_{0}-4 h_{0}\right) \tilde{\xi}_{6}\right)\right]
\end{aligned}
$$

where we remind the reader that a prime denotes a derivative with respect to $r$ not $y(2.33)$.

\subsection{2 $\phi^{a}$ equations}

The $\phi_{a}$ equations benefit from a field redefinition as well,

$$
\begin{aligned}
& \phi^{a}=(\alpha, \beta, \gamma, z, f, h), \\
& \tilde{\phi}_{a}=\left(\phi_{1}-\phi_{2}, \phi_{1}+\phi_{2}-2 \phi_{3}, \phi_{3}, \phi_{4}, \phi_{5}, \phi_{6}\right)
\end{aligned}
$$


and we find

$$
\begin{gathered}
\tilde{\phi}_{1}^{\prime}=\frac{1}{12} e^{-3\left(\alpha_{0}+\beta_{0}\right)}\left[-3 \tilde{\xi}_{1}+4 \tilde{\xi}_{2}+3\left(\tilde{\xi}_{3}-4 e^{2\left(\alpha_{0}+\beta_{0}\right)}\left(e^{2 \alpha_{0}}+e^{2 \beta_{0}}\right) \tilde{\phi}_{1}\right)\right] \\
\tilde{\phi}_{2}^{\prime}=\frac{1}{12} e^{-3\left(\alpha_{0}+\beta_{0}\right)}\left[-3 \tilde{\xi}_{1}+7 \tilde{\xi}_{3}+12 e^{2\left(\alpha_{0}+\beta_{0}\right)}\left(3\left(e^{2 \beta_{0}}-e^{2 \alpha_{0}}\right) \tilde{\phi}_{1}-4 e^{2 \gamma_{0}} \tilde{\phi}_{2}\right)\right] \\
\tilde{\phi}_{3}^{\prime}=\frac{1}{12} e^{-3\left(\alpha_{0}+\beta_{0}\right)}\left[\tilde{\xi}_{1}-3\left(\tilde{\xi}_{3}+6 e^{2\left(\alpha_{0}+\beta_{0}\right)}\left(\left(e^{2 \beta_{0}}-e^{2 \alpha_{0}}\right) \tilde{\phi}_{1}-e^{2 \gamma_{0}} \tilde{\phi}_{2}\right)\right)\right] \\
\tilde{\phi}_{5}^{\prime}=\frac{2}{m^{2}} e^{-3\left(\alpha_{0}-\beta_{0}\right)}\left[e^{3 z_{0}} \tilde{\xi}_{5}+3 m^{2}\left(3 h_{0} \tilde{\phi}_{1}-\tilde{\phi}_{6}\right)\right] \\
\tilde{\phi}_{6}^{\prime}=\frac{1}{6 m^{2}} e^{\alpha_{0}-\beta_{0}}\left[e^{3 z_{0}} \tilde{\xi}_{6}-3 m^{2}\left(f_{0} \tilde{\phi}_{1}-4 h_{0} \tilde{\phi}_{1}+\tilde{\phi}_{5}-4 \tilde{\phi}_{6}\right)\right] \\
\tilde{\phi}_{4}^{\prime}=\frac{1}{9} e^{-3\left(\alpha_{0}+\beta_{0}+z_{0}\right)}\left[2 e^{3 z_{0}} \tilde{\xi}_{4}+m^{2}\left(\left[1-54 h_{0}\left(f_{0}-2 h_{0}\right)\right] \tilde{\phi}_{4}+18 f_{0} \tilde{\phi}_{6}\right.\right. \\
\left.\left.\quad+\tilde{\phi}_{2}+2 \tilde{\phi}_{3}+18 h_{0}\left[\tilde{\phi}_{5}-4 \tilde{\phi}_{6}-3\left(f_{0}-2 h_{0}\right)\left(\tilde{\phi}_{2}+2 \tilde{\phi}_{3}\right)\right]\right)\right]
\end{gathered}
$$

\section{The force on a probe M2}

Before solving the above equations, we compute the force on a probe M2-brane in the perturbed solution space. As was found in the analogous IIB scenario [22], the force turns out to benefit from remarkable cancellations and is ultimately quite simple.

The membrane action for a probe M2 brane (which by abusing notation we refer to as the DBI action) is

$$
\begin{aligned}
V^{D B I} & =\sqrt{-g_{00} g_{11} g_{22}}, \\
& =e^{-3 z}
\end{aligned}
$$

and, in the first-order approximation, its derivative with respect to $r$ is

$$
F^{D B I}=-\frac{d V_{0}^{D B I}}{d r}+3 e^{-3 z_{0}}\left(\tilde{\phi}_{4}^{\prime}-3 z_{0}^{\prime} \tilde{\phi}_{4}\right)
$$

We next consider the derivative of the WZ action with respect to $r$, which gives the force exerted on the M2-brane by the $G^{(4)}$ field:

$$
\begin{aligned}
F^{W Z} & =-\frac{d V^{W Z}}{d r} \\
& =G_{012 r}^{(4)} \\
& =-6 m^{2}\left[h(f-2 h)-\frac{1}{54}\right] e^{-3(\alpha+\beta)-6 z} .
\end{aligned}
$$

The zeroth-order and first-order WZ forces thus are

$$
F_{0}^{W Z}=-6 m^{2}\left[h_{0}\left(f_{0}-2 h_{0}\right)-\frac{1}{54}\right] e^{-3\left(\alpha_{0}+\beta_{0}\right)-6 z_{0}}
$$


and

$$
\begin{aligned}
F_{1}^{W Z}=-6 m^{2}[ & h_{0}\left(\tilde{\phi}_{5}-2 \tilde{\phi}_{6}\right)+\tilde{\phi}_{6}\left(f_{0}-2 h_{0}\right) \\
& \left.-3\left(\tilde{\phi}_{2}+2 \tilde{\phi}_{3}+2 \tilde{\phi}_{4}\right)\left(h_{0}\left(f_{0}-2 h_{0}\right)-\frac{1}{54}\right)\right] e^{-3\left(\alpha_{0}+\beta_{0}\right)-6 z_{0}} .
\end{aligned}
$$

Combining these two contributions to the force we see that the zeroth-order contributions cancel as expected. Then using the explicit $\phi^{a}$ equations from section 2.4.2 we find the beautiful result

$$
\begin{aligned}
F & =F_{1}^{D B I}+F_{1}^{W Z} \\
& =\frac{2}{3} e^{-3\left(\alpha_{0}+\beta_{0}+z_{0}\right)(r)} \tilde{\xi}_{4}(r) .
\end{aligned}
$$

At this point it is worthwhile to preemptively trumpet the result (4.3) from section 4 where the exact solution for the mode $\tilde{\xi}_{4}$ is found:

$$
\begin{aligned}
F & =\frac{2}{3} e^{-3\left(\alpha_{0}+\beta_{0}\right)(r)} Z_{0} X_{4} \\
& =\frac{18 Z_{0} X_{4}}{(2+\cosh 2 r)^{3 / 4} \sinh ^{3} r},
\end{aligned}
$$

where $Z_{0}$ is some numerical factor which we found convenient not to absorb into the $X_{4}$ integration constant,

$$
Z_{0} \equiv e^{-3 z_{0}(0)}
$$

So, the UV expansion of the force felt by a probe M2 brane in the first-order perturbed solution is always

$$
F_{r} \sim X_{4} e^{-9 r / 2}+\mathcal{O}\left(e^{-17 r / 2}\right)
$$

In terms of $\rho$, the "standard" radial coordinate, ${ }^{5}$ this force comes from a potential proportional to $\rho^{-6}$, which agrees with a straightforward extension of the brane-antibrane force analysis of [23] to this system. This will be further discussed in a forthcoming publication [46].

\section{The space of solutions}

In this section we find the generic solution to the system (2.37)-(2.50). This solution space has twelve integration constants of which ten are physical. We have managed to solve the $\tilde{\xi}_{a}$ equations exactly whereas for the $\phi_{a}$ equations we have resorted to solving them in the IR and UV limits.

\footnotetext{
${ }^{5}$ Related to $r$ via $\cosh (2 r) \sim \rho^{8 / 3}$.
} 


\subsection{Analytic solutions for the $\tilde{\xi}$ 's}

The first equation (2.37) is solved by

$$
\tilde{\xi}_{4}=X_{4} \exp \left(6 m^{2} \int_{0}^{r} d r^{\prime} e^{-3\left(\alpha_{0}+\beta_{0}+z_{0}\right)}\left[\left(f_{0}-2 h_{0}\right) h_{0}-\frac{1}{54}\right]\right),
$$

which appears to be a double integral. However, using a standard notation for the warp factor $H_{0}=e^{3 z_{0}}$, since we have

$$
\frac{d H_{0}}{d r}=-2^{3} 3 m^{2} \frac{e^{2 \gamma_{0}}}{\sinh ^{3} 2 r} \tanh ^{4} r
$$

we actually find

$$
\begin{aligned}
\tilde{\xi}_{4} & =X_{4} \exp \left(\int_{0}^{r} d r^{\prime} \frac{1}{H_{0}} \frac{d H_{0}}{d r^{\prime}}\right), \\
& =X_{4} e^{3\left(z_{0}(r)-z_{0}(0)\right)} .
\end{aligned}
$$

It immediately follows that

$$
\tilde{\xi}_{1}=X_{1}+2 X_{4} e^{3\left(z_{0}(r)-z_{0}(0)\right)} .
$$

We find convenient not to include $e^{-3 z_{0}(0)}$ into the integration constant $X_{4}$, and will use the notation

$$
Z_{0} \equiv e^{-3 z_{0}(0)}
$$

We were also able to find exact analytic expressions for $\tilde{\xi}_{3}$ and $\tilde{\xi}_{5,6}$, in term of $y^{4} \equiv$ $2+\cosh (2 r):$

$$
\begin{aligned}
\tilde{\xi}_{3}= & y^{4}\left(y^{4}-3\right)^{2} X_{3}-\frac{m^{2} Z_{0} X_{4}}{18 \sqrt{2}} \frac{y\left(y^{4}-3\right)}{\left(y^{4}-1\right)^{3 / 2}}\left[-96+599 y^{4}-550 y^{8}+119 y^{12}\right. \\
& -y^{3} \sqrt{y^{4}-1}\left(3-4 y^{4}+y^{8}\right)\left(163 F\left(\arcsin \left(\frac{1}{y}\right) \mid-1\right)\right. \\
& \left.\left.+22\left[\Pi\left(-\sqrt{3} ;-\arcsin \left(\frac{1}{y}\right) \mid-1\right)+\Pi\left(\sqrt{3} ;-\arcsin \left(\frac{1}{y}\right) \mid-1\right)\right]\right)\right],
\end{aligned}
$$

where $F(\phi \mid q)$ is given in $(2.35)$ and $\Pi(n ; \phi \mid m)$ is an incomplete elliptic integral of the third kind

$$
\Pi(n ; \phi \mid m)=\int_{0}^{\phi} \frac{d \theta}{\left(1-n \sin (\theta)^{2}\right) \sqrt{1-m \sin (\theta)^{2}}} .
$$


The expressions for $\tilde{\xi}_{5,6}$ are as follows:

$$
\begin{aligned}
& \tilde{\xi}_{5}=\frac{1}{4 \sqrt{2}\left(y^{4}-3\right) \sqrt{y^{4}-1}}\left[\sqrt{6} Z_{0} X_{4} m^{2} y\left(13-11 y^{4}\right) \sqrt{y^{4}-1}\right. \\
& +4\left[\left(y^{4}-1\right)^{2} X_{5}+\left(y^{4}-3\right)\left(1+y^{4}\right) X_{6}\right] \\
& +\sqrt{6} Z_{0} m^{2} X_{4}\left[\left(19+7 y^{4}\left(y^{4}-2\right)\right) F\left(\arcsin \left(\frac{1}{y}\right) \mid-1\right)\right. \\
& -2\left(y^{4}-3\right)\left(1+y^{4}\right)\left(\Pi\left(-\sqrt{3} ;-\arcsin \left(\frac{1}{y}\right) \mid-1\right)\right. \\
& \left.\left.\left.+\Pi\left(\sqrt{3} ;-\arcsin \left(\frac{1}{y}\right) \mid-1\right)\right)\right]\right] \text {, } \\
& \tilde{\xi}_{6}=\frac{\sqrt{2}}{\left(y^{4}-3\right)\left(y^{4}-1\right)^{3 / 2}}\left[( y ^ { 4 } - 7 ) ( y ^ { 4 } - 1 ) ^ { 2 } \left[X_{5}+\sqrt{\frac{3}{2}} Z_{0} m^{2} X_{4}\left(\frac{7 y-5 y^{5}}{\left(y^{4}-1\right)^{3 / 2}}\right.\right.\right. \\
& \left.\left.+5 F\left(\arcsin \left(\frac{1}{y}\right) \mid-1\right)\right)\right]+\frac{1}{4}\left(y^{4}-3\right)^{2}\left[-\sqrt{6} Z_{0} m^{2} X_{4} y \sqrt{y^{4}-1}\right. \\
& +4\left(y^{4}-3\right) X_{6}-\sqrt{6} Z_{0} m^{2} X_{4}\left(y^{4}-3\right)\left(3 F\left(\arcsin \left(\frac{1}{y}\right) \mid-1\right)\right. \\
& \left.\left.\left.+2\left(\Pi\left(-\sqrt{3} ;-\arcsin \left(\frac{1}{y}\right) \mid-1\right)+\Pi\left(\sqrt{3} ;-\arcsin \left(\frac{1}{y}\right) \mid-1\right)\right)\right)\right]\right] .
\end{aligned}
$$

Lastly, $\tilde{\xi}_{2}$ is given by the zero-energy condition (2.10) but its explicit form does not appear to be too enlightening. In appendix B we provide the IR and UV series expansions of the above solutions for $\tilde{\xi}^{i}$.

\subsection{Solving the $\phi^{i}$ equations}

\subsubsection{The space of solutions}

We now solve the system of equations for $\phi^{i}(2.45)-(2.49)$ using the Lagrange method of variation of parameters.

Equation (2.45) is solved by

$$
\tilde{\phi}_{1}=\frac{\tilde{\lambda}^{1}(r)}{\sinh (2 r)}
$$

with

$$
\tilde{\lambda}^{1}=\frac{9}{2} \int \frac{\cosh (r)}{\sinh (r)^{2}(2+\cosh (2 r))^{3 / 4}}\left[-3 \tilde{\xi}_{1}+4 \tilde{\xi}_{2}+3 \tilde{\xi}_{3}\right]+Y_{1}^{I R} .
$$

$\tilde{\xi}_{2}$ and $\tilde{\xi}_{3}$ are given in section 4.1 above and $\sinh (2 r)^{-1}$ is the homogeneous solution to the $\tilde{\phi}_{1}$ equation.

The same Lagrange method is used for $\tilde{\phi}_{2}$, which is given by

$$
\tilde{\phi}_{2}=\frac{\tilde{\lambda}^{2}(r)}{\sinh (r)^{4}(2+\cosh (2 r))},
$$


where

$$
\begin{aligned}
\tilde{\lambda}^{2}= & \frac{9}{4} \int \sinh (r)(2+\cosh (2 r))^{1 / 4}\left[-3 \tilde{\xi}_{1}+7 \tilde{\xi}_{3}-\frac{4}{3} \frac{\sinh (r)^{2}}{\cosh (r)}(2+\cosh (2 r))^{3 / 4} \tilde{\phi}_{1}\right] \\
& +Y_{2}^{I R} .
\end{aligned}
$$

From this, we obtain an integral expression for $\tilde{\phi}_{3}$ :

$$
\tilde{\phi}_{3}=\frac{9}{4} \int \frac{\left[\tilde{\xi}_{1}-3 \tilde{\xi}_{3}+\frac{2}{3} \frac{\sinh (r)^{2}}{\cosh (r)}(2+\cosh (2 r))^{3 / 4} \tilde{\phi}_{1}+2 \frac{\sinh (r)^{2} \cosh (r)^{3}}{(2+\cosh (2 r))^{1 / 4}} \tilde{\phi}_{2}\right]}{\sinh (r)^{3}(2+\cosh (2 r))^{3 / 4}}+Y_{3}^{I R}
$$

The fluxes $\left(\tilde{\phi}_{5}, \tilde{\phi}_{6}\right)=(f, h)$ are given by

$$
\left(\begin{array}{c}
\tilde{\phi}_{5} \\
\tilde{\phi}_{6}
\end{array}\right)=\left(\begin{array}{cc}
\cosh (r)^{3} \tanh (r)^{6} & \cosh (r)^{3}\left[2-3 \tanh (r)^{2}\right] \\
\frac{1}{2}\left[\operatorname{sech}(r)-\cosh (r)^{3}\right] & \frac{1}{2} \cosh (r)^{3}
\end{array}\right)\left(\begin{array}{c}
\tilde{\lambda}_{5} \\
\tilde{\lambda}_{6}
\end{array}\right),
$$

where the derivatives of $\tilde{\lambda}_{5}$ and $\tilde{\lambda}_{6}$ are given by

$$
\left(\begin{array}{c}
\tilde{\lambda}_{5}^{\prime} \\
\tilde{\lambda}_{6}^{\prime}
\end{array}\right)=\left(\begin{array}{cc}
\frac{1}{4} \cosh (r) \operatorname{coth}(r)^{2} & \frac{1}{2}[\cosh (r)-2 \operatorname{coth}(r) \operatorname{csch}(r)] \\
\frac{1}{8}[3+\cosh (2 r)] \operatorname{sech}(r) & \frac{1}{2} \sinh (r) \tanh (r)^{3}
\end{array}\right)\left(\begin{array}{c}
b_{5} \\
b_{6}
\end{array}\right),
$$

and $b_{5}, b_{6}$ are the right-hand side of (2.48) and (2.49) respectively. The $2 \times 2$ matrix appearing in (4.16) is the inverse of the matrix of homogeneous solutions written in (4.15). We will call $Y_{5}$ and $Y_{6}$ the constants arising from integrating (4.16), even though the two functions $\tilde{\phi}_{5}$ and $\tilde{\phi}_{6}$ depend on both of them.

Finally, relying on the same method, the equation for $\tilde{\phi}_{4}$ is solved to

$$
\tilde{\phi}_{4}=e^{-3 z_{0}(r)} \tilde{\lambda}_{4}, \quad \tilde{\lambda}_{4}=\int e^{3 z_{0}(r)} b_{4}(r)+Y_{4}^{I R},
$$

where $b_{4}(r)$ is the right-hand side of $(2.50)$ (setting $\tilde{\phi}_{4}$ to zero).

\subsubsection{IR behavior}

We now give the IR expansions of the $\phi^{i}$ s. We only write the divergent and constant terms since terms which are regular in the IR do not provide any constraint on our solution space. $Z_{0}$ is defined in (3.7). The $X_{i}$ integration constants are those appearing in the exact solutions for the $\tilde{\xi}_{i}$ 's $(4.3)-(4.9)$ :

$$
\begin{aligned}
\tilde{\phi}_{1}= & -\frac{1}{r^{2}}\left[\frac{27 X_{1}+30 X_{4}-16 \sqrt{3} X_{5}}{43^{3 / 4}}\right]+\frac{1}{2 r} Y_{1}^{I R} \\
& +\left[\frac{189 X_{1}+\left(498-1983^{1 / 4} Z_{0} m^{2}\right) X_{4}+80 \sqrt{3} X_{5}}{123^{3 / 4}}\right]+\mathcal{O}(r),
\end{aligned}
$$




$$
\begin{aligned}
\tilde{\phi}_{2}= & \frac{Y_{2}^{I R}}{3 r^{4}}+\frac{1}{r^{2}}\left[\frac{9}{4} 3^{1 / 4} X_{1}+\frac{3}{2} 3^{1 / 4} X_{4}-2 \sqrt{3} 3^{1 / 4} X_{5}-\frac{4}{9} Y_{2}^{I R}\right]-\frac{1}{2 r} Y_{1}^{I R} \\
& -\left[63^{1 / 4} X_{1}+\frac{23}{2} 3^{1 / 4} X_{4}-6 \sqrt{3} Z_{0} m^{2} X_{4}-\frac{1}{3^{1 / 4}} X_{5}-\frac{41}{135} Y_{2}^{I R}\right] \\
& +\mathcal{O}(r), \\
\tilde{\phi}_{3}= & -\frac{Y_{2}^{I R}}{8 r^{4}}-\frac{1}{r^{2}}\left[\frac{93^{1 / 4} X_{1}-123^{3 / 4} X_{5}-4 Y_{2}^{I R}}{24}\right] \\
& +\left[Y_{3}^{I R}+\frac{3^{1 / 4}}{8}\left(-183^{1 / 4} Z_{0} m^{2} X_{4}+21 X_{1}+48 X_{4}+4 \sqrt{3} X_{5}\right) \log (r)\right] \\
& +\mathcal{O}(r), \\
\tilde{\phi}_{4}= & -\frac{1}{r^{2}}\left[\frac{18 X_{4}-4 \sqrt{3} X_{5}+Z_{0} m^{2}\left(Y_{2}^{I R}-24 \sqrt{3} Y_{6}^{I R}\right)}{83^{3 / 4}}\right] \\
& -\left[\frac{1}{4}\left(Z_{0} m^{2}\left(\frac{3 \sqrt{3}}{2} X_{4}-X_{5}\right)-4 Z_{0} Y_{4}^{I R}\right)\right. \\
& +\frac{1}{48} Z_{0}^{2} m^{4}\left(\sqrt{3} Y_{2}^{I R}-72 Y_{6}^{I R}\right)+\left[\frac{3}{2} 3^{1 / 4} X_{4}-\frac{X_{5}}{3^{1 / 4}}\right. \\
& \left.\left.+\frac{1}{36} Z_{0} m^{2}\left(81 \sqrt{3} X_{1}+78 \sqrt{3} X_{4}-168 X_{5}+113^{1 / 4} Y_{2}^{I R}-723^{3 / 4} Y_{6}^{I R}\right)\right] \log (r)\right] \\
& +\mathcal{O}(r), \\
& +\left[\frac{3^{3 / 4}}{16} X_{1}-\frac{1}{18} \frac{X_{5}+\frac{\sqrt{3}}{2} X_{4}}{Z_{0} m^{2}}-\frac{7}{72} 3^{3 / 4} X_{4}-\frac{5}{18} 3^{1 / 4} X_{5}+\frac{1}{2} Y_{6}^{I R}\right]+\mathcal{O}(r) \\
\tilde{\phi}_{5}= & 2 Y_{6}^{I R}+\left[\frac{9}{8} 3^{3 / 4} X_{1}+\frac{3}{4} 3^{3 / 4} X_{4}-23^{1 / 4} X_{5}+\frac{1}{2 Z_{0} m^{2}}\left(X_{5}+\frac{\sqrt{3}}{2} X_{4}\right)\right] r^{2}+\mathcal{O}\left(r^{3}\right), \\
\tilde{\phi}_{6}= & \frac{1}{r^{2}} \frac{X_{5}+\frac{\sqrt{3}}{6} X_{4} m^{2}}{(4.21)} \\
& =(4.22) \\
& \\
&
\end{aligned}
$$

Note that in the $\tilde{\phi}_{5}$ expansion we have also displayed the term of order $r^{2}$ - this term will be relevant for the singularity analysis in section 6 .

\subsubsection{UV behavior}

We provide the UV asymptotics for all six $\tilde{\phi}_{i}$ 's, incorporating terms which decay not faster than $e^{-13 r / 2}$. However, as appears in table 1 below, a few modes have leading behavior in the UV which is even more convergent than this.

$$
\begin{aligned}
\tilde{\phi}_{1}= & \frac{18}{2^{1 / 4}} X_{3} e^{-r / 2}+2 Y_{1}^{U V} e^{-2 r}-42^{3 / 4}\left[\frac{27}{2} X_{1}-27 X_{3}+8 \sqrt{3}\left(X_{5}+X_{6}\right)\right] e^{-5 r / 2} \\
& -\left[\frac{1089}{102^{1 / 4}} X_{3}-\frac{128}{5} 2^{3 / 4} \sqrt{3}\left(X_{5}+X_{6}\right)\right] e^{-9 r / 2}+2 Y_{1}^{U V} e^{-6 r} \\
& +\mathcal{O}\left(e^{-13 r / 2}\right)
\end{aligned}
$$




$$
\begin{aligned}
& \tilde{\phi}_{2}=\frac{21}{52^{1 / 4}} X_{3} e^{3 r / 2}-\frac{17523}{1402^{1 / 4}} e^{-5 r / 2} X_{3}-12 Y_{1}^{U V} e^{-4 r} \\
& +42^{3 / 4}\left[99 X_{1}-\frac{1719}{10} X_{3}+64 \sqrt{3}\left(X_{5}+X_{6}\right)\right] e^{-9 r / 2}+32 Y_{2}^{U V} e^{-6 r} \\
& +\mathcal{O}\left(e^{-13 r / 2}\right) \text {, } \\
& \tilde{\phi}_{3}=-\frac{27}{102^{1 / 4}} X_{3} e^{3 r / 2}+Y_{3}^{U V}+\frac{9693}{2802^{1 / 4}} X_{3} e^{-5 r / 2}+\frac{15}{4} Y_{1}^{U V} e^{-4 r} \\
& -2^{3 / 4}\left[130 X_{1}-\frac{1113}{5} X_{3}+\frac{256}{\sqrt{3}}\left(X_{5}+X_{6}\right)\right] e^{-9 r / 2}-12 Y_{2}^{U V} e^{-6 r} \\
& +\mathcal{O}\left(e^{-13 r / 2}\right) \text {, } \\
& \tilde{\phi}_{4}=\frac{3}{162^{3 / 4}} \frac{Y_{4}^{U V}}{m^{2}} e^{9 r / 2}+\frac{27}{262^{3 / 4}} \frac{Y_{4}^{U V}}{m^{2}} e^{5 r / 2}+\frac{9}{52^{1 / 4}} X_{3} e^{3 r / 2}+\frac{350271}{1838722^{3 / 4}} \frac{Y_{4}^{U V}}{m^{2}} e^{r / 2} \\
& -2\left[Y_{3}^{U V}+\sqrt{3}\left(Y_{5}^{U V}-Y_{6}^{U V}\right)\right]+\frac{216}{325} 2^{3 / 4} X_{3} e^{-r / 2}+\frac{484605}{2987922^{3 / 4}} \frac{Y_{4}^{U V}}{m^{2}} e^{-3 r / 2} \\
& +\frac{144}{13} \sqrt{3} Y_{6}^{U V} e^{-2 r}+\frac{3985953003}{140777002^{1 / 4}} X_{3} e^{-5 r / 2}+\frac{7978373883}{211305702402^{3 / 4}} \frac{Y_{4}^{U V}}{m^{2}} e^{-7 r / 2} \\
& +\left[\frac{273}{34} Y_{1}^{U V}+\frac{78912 \sqrt{3}}{2873} Y_{6}^{U V}\right] e^{-4 r} \\
& -2^{3 / 4}\left[4 \frac{229}{5} X_{1}-\frac{1707341851}{2691325} X_{3}+4 \frac{256}{3 \sqrt{3}}\left(X_{5}+X_{6}\right)\right] e^{-9 r / 2} \\
& +\frac{473729599251}{9957781225602^{3 / 4}} \frac{Y_{4}^{U V}}{m^{2}} e^{-11 r / 2}+\mathcal{O}\left(e^{-6 r}\right), \\
& \tilde{\phi}_{5}=\frac{1}{8}\left(Y_{5}^{U V}-Y_{6}^{U V}\right) e^{3 r}-\frac{9}{8}\left(Y_{5}^{U V}-Y_{6}^{U V}\right) e^{r}+\frac{1}{8}\left(39 Y_{5}^{U V}+9 Y_{6}^{U V}\right) e^{-r} \\
& +19 \frac{42^{3 / 4}}{\sqrt{3}} X_{3} e^{-3 r / 2}+\left[\frac{14}{3 \sqrt{3}} Y_{1}^{U V}-\frac{1}{8}\left(111 Y_{5}^{U V}+Y_{6}^{U V}\right)\right] e^{-3 r} \\
& -42^{3 / 4}\left[2 \frac{279}{65} \sqrt{3} X_{1}+\frac{147}{65} \sqrt{3} X_{3}+2 \frac{308}{39}\left(X_{5}+X_{6}\right)\right] e^{-7 r / 2} \\
& +10\left[-\frac{2}{\sqrt{3}} Y_{1}^{U V}+3 Y_{5}^{U V}\right] e^{-5 r} \\
& +\frac{56}{1105} 2^{3 / 4}\left[3071 \sqrt{3} X_{1}-\frac{166409 \sqrt{3}}{56} X_{3}+\frac{18716}{3}\left(X_{5}+X_{6}\right)\right] e^{-11 r / 2} \\
& +\mathcal{O}\left(e^{-13 r / 2}\right) \text {, } \\
& \tilde{\phi}_{6}=-\frac{1}{16}\left(Y_{5}^{U V}-Y_{6}^{U V}\right) e^{3 r}-\frac{3}{16}\left(Y_{5}^{U V}-Y_{6}^{U V}\right) e^{r}+\frac{1}{16}\left(13 Y_{5}^{U V}+3 Y_{6}^{U V}\right) e^{-r} \\
& +\frac{10}{\sqrt{3}} 2^{3 / 4} X_{3} e^{-3 r / 2}+\left[\frac{1}{3 \sqrt{3}} Y_{1}^{U V}-\frac{1}{16}\left(17 Y_{5}^{U V}-Y_{6}^{U V}\right)\right] e^{-3 r} \\
& -42^{3 / 4}\left[\frac{33}{65} \sqrt{3} X_{1}+\frac{9 \sqrt{3}}{130} X_{3}+\frac{116}{117}\left(X_{5}+X_{6}\right)\right] e^{-7 r / 2} \\
& -\left[\frac{2}{3 \sqrt{3}} Y_{1}^{U V}-Y_{5}^{U V}\right] e^{-5 r}
\end{aligned}
$$




\begin{tabular}{|c|c|c|}
\hline $\operatorname{dim} \Delta$ & non-norm $/$ norm & int. constant \\
\hline 6 & $\rho_{\text {AdS }}^{3} / \rho_{\text {AdS }}^{-6}$ & $Y_{4}^{U V} / X_{4}$ \\
\hline 5 & $\rho_{\text {AdS }}^{2} / \rho_{\text {AdS }}^{-5}$ & $Y_{5}^{U V}-Y_{6}^{U V} / X_{5}-X_{6}$ \\
\hline 4 & $\rho_{\text {AdS }} / \rho_{\text {AdS }}^{-4}$ & $X_{3} / Y_{2}^{U V}$ \\
\hline 3 & $\rho_{\text {AdS }}^{0} / \rho_{\text {AdS }}^{-3}$ & $Y_{3} / X_{2}$ \\
\hline $7 / 3$ & $\rho_{\text {AdS }}^{-2 / 3} / \rho_{\text {AdS }}^{-7 / 3}$ & $Y_{5}^{U V}+Y_{6}^{U V} / X_{5}+X_{6}$ \\
\hline $5 / 3$ & $\rho_{\text {AdS }}^{-4 / 3} / \rho_{\text {AdS }}^{-5 / 3}$ & $Y_{1}^{U V} / X_{1}$ \\
\hline
\end{tabular}

Table 1. The UV behavior of the twelve $\mathrm{SO}(5)$-invariant modes in the deformation space of the CGLP solution. As discussed below, only ten of these modes are physical, and the mode of dim. 3 is a gauge artifact.

$$
\begin{aligned}
& +\frac{4}{1105 \sqrt{3}} 2^{3 / 4}\left[3713 X_{1}-\frac{30221}{8} X_{3}+2932 \sqrt{3}\left(X_{5}+X_{6}\right)\right] e^{-11 r / 2} \\
& +\mathcal{O}\left(e^{-13 r / 2}\right) .
\end{aligned}
$$

To understand the holographic physics of the $\tilde{\phi}^{i}$ modes, we tabulate the leading UV behavior coming from each mode. To each local operator $\mathcal{O}_{i}$ of quantum dimension $\Delta$ in the field theory, the holographic dictionary associates two modes in the dual AdS space, one normalizable and one non-normalizable $[47,48]$. These two supergravity modes are dual respectively to the vacuum expectation value (VEV) $\left\langle 0\left|\mathcal{O}_{i}\right| 0\right\rangle$ and the deformation of the action $\delta S \sim \int d^{d} x \mathcal{O}_{i}$ :

$$
\begin{aligned}
& \text { normalizable modes } \sim \rho_{\text {AdS }}^{-\Delta} \leftrightarrow \text { field theory VEV's } \\
& \text { non-normalizable modes } \sim \rho_{\text {AdS }}^{\Delta-3} \leftrightarrow \text { field theory deformations of the action } .
\end{aligned}
$$

Here we refer to the standard AdS radial coordinate $\rho_{\text {AdS }}$, to be distinguished from the radial coordinate on the cone, $\rho$. In the UV, we have $\rho \sim e^{3 r / 4}$ and $\rho_{\text {AdS }} \sim \rho^{2} / m^{1 / 3}$ with the factor of $m^{1 / 3}$ taken with respect to the conventions of [18].

In table 1 we have summarized which integration constants correspond to normalizable and non-normalizable modes. As stated in a previous section, the $X_{i}$ are integration constants for the $\xi_{i}$ modes and break supersymmetry, while the $Y_{i}$ are integration constants for the modes $\phi^{i}$. It is very interesting to note that in all cases a normalizable/nonnormalizable pair consists of one BPS mode and one non-BPS mode.

As already mentioned, the mode $\tilde{\xi}_{4}$, whose integration constant is $X_{4}$ and which is the only mode accountable for the force felt by a probe M2-brane in the first-order perturbation to the CGLP background [20], is the most convergent mode in the UV, though this cannot be seen from the expansions we have provided but is apparent at higher order in the asymptotics that we have computed.

Taking into account a rescaling which culls $Y_{3}$ and the zero energy condition which eliminates $X_{2}$, we are left with a total of ten integration constants or five modes. The absence of a physical mode behaving as $\rho_{\text {AdS }}^{0}$ is related to the quantization of the level of the Chern-Simons matter theory. This is unlike in four-dimensional gauge theories, 
where we expect a dimension-four operator corresponding to the dilaton. Note also that in table 1 we see explicitly the dimension $\Delta=7 / 3$ operator discussed in [18]. We have been somewhat glib in writing $X_{5}-X_{6}$ or $Y_{5}+Y_{6}$. The numerical factors in the combination of those integration constants are actually different, but can be rescaled to the shorthand notation we use.

\section{Boundary conditions for M2 branes}

Within the space of solutions that we have derived in section 4 we now proceed to find the modes which arise from the backreaction of a set of anti-M2 branes smeared on the finite-sized $S^{4}$ at the tip of the Stenzel-CGLP solution $(r=0)$. For describing them it is necessary to carefully impose the correct infrared boundary conditions.

The gravity solution for a stack of localized M2-branes in flat space has a warp factor $H(\rho)=1+Q / \rho^{6}$ and as $\rho \rightarrow 0$ the full solution is smooth due to the infinite throat. However when these branes are smeared in $n$-dimensions, the warp factor scales as $\rho^{-6+n}$ as $\rho \rightarrow 0$ since it is now the solution to a wave equation in dimension $d=8-n$. This is the IR boundary condition that we will impose on the solution.

We must furthermore bring to bear appropriate boundary conditions on the various fluxes. This is rather simple for M2 branes in flat space, where the energy from $G^{(4)}$ is the same as that from the curvature. In the presence of other types of flux, the IR boundary conditions are more intricate. When the background is on-shell, contributions to the stress tensor from all types of flux taken together cancel the energy from the curvature: this is the basic nature of Einstein's equation but this is too wobbly a criterion to signal the presence of M2 branes. Instead, the right set of boundary conditions for M2 branes should enforce that the dominant contribution to the stress-energy tensor comes from the $G^{(4)}$ flux.

\subsection{BPS M2 branes}

The M2 brane charge varies with the radial coordinate $r$ of a section of the Stenzel space [19]:

$$
\begin{aligned}
\mathcal{Q}_{M 2}(r) & =\frac{1}{\left(2 \pi \ell_{p}\right)^{6}} \int_{\mathcal{M}_{7}} \star G_{4}, \\
& =-\frac{6 m^{2} \operatorname{Vol}\left(V_{5,2}\right)}{\left(2 \pi \ell_{p}\right)^{6}}\left(h_{0}(r)\left(f_{0}(r)-2 h_{0}(r)\right)-\frac{1}{54}\right),
\end{aligned}
$$

with $\ell_{p}$ the Planck length in eleven dimensions, $\mathcal{M}_{7}$ a constant $r$ section of the transverse Stenzel space of volume $\operatorname{Vol}\left(V_{5,2}\right)=\frac{27 \pi^{4}}{128}$ [49]. The number of units of $G_{4}$ flux through the $S^{4}$ is

$$
\begin{aligned}
q(r) & =\frac{1}{\left(2 \pi \ell_{p}\right)^{3}} \int_{S^{4}} G_{4}, \\
& =-\frac{16 \pi^{2} m}{\left(2 \pi \ell_{p}\right)^{3}} h_{0}(r) .
\end{aligned}
$$


In the smooth solution their IR values $(r \rightarrow 0)$ are

$$
\mathcal{Q}_{M 2}^{I R}=0, \quad q^{I R}=\frac{1}{\left(2 \pi \ell_{p}\right)^{3}} \frac{8 \pi^{2} m}{3^{3 / 2}},
$$

reflecting the fact that all M2 charge is dissolved in fluxes. One can obtain a BPS solution in which smeared M2 branes are added at the tip of the Stenzel space [19] simply by shifting $\star G_{4}$ in such a way that $f-4 h$ does not change. ${ }^{6}$ Under shifts of $f \rightarrow f+2 N$ and $h \rightarrow h+\frac{N}{2}$, the IR M2 brane charge changes to

$$
\mathcal{Q}_{M 2} \rightarrow \mathcal{Q}_{M 2}+\Delta \mathcal{Q}_{M 2},
$$

where we define

$$
\Delta \mathcal{Q}_{M 2}=-\frac{6 m^{2} \operatorname{Vol}\left(V_{5,2}\right)}{\left(2 \pi \ell_{p}\right)^{6}}\left(\frac{1}{2} N^{2}-\frac{2}{3^{3 / 2}} N\right)
$$

whereas the variation in the units of flux through the $S^{4}$ amounts to $\frac{8 \pi^{2} m N}{\left(2 \pi \ell_{p}\right)^{3}}$. This introduces in the IR a $-\Delta \mathcal{Q}_{M 2} / r^{2}$ singularity in the warp factor

$$
H_{0}(r)=162 m^{2} \int^{r} \frac{h_{0}\left(f_{0}-2 h_{0}\right)-\frac{1}{54}}{\sinh \left(r^{\prime}\right)^{3}\left(2+\cosh \left(2 r^{\prime}\right)\right)^{3 / 4}} d r^{\prime} .
$$

This singularity is to be expected as we have smeared BPS M2 branes (whose harmonic function diverges as $1 / r^{6}$ near the sources) on the $S^{4}$ of the transverse space. It is interesting to see how this BPS solution arises in the first-order expansion around the BPS CGLP background [20] in the context of our perturbation apparatus. Given that the $\xi^{i}$ modes are associated to supersymmetry-breaking, all the $X_{i}$ must be set to zero:

$$
X_{i}=0
$$

Since all the $\tilde{\xi}^{i}$ are zero,

$$
Y_{1}^{I R}=Y_{1}^{U V} .
$$

In the IR and the UV, $e^{z_{0}+2 \alpha_{0}}, e^{z_{0}+2 \beta_{0}}$ and $e^{z_{0}+2 \gamma_{0}}$ do not blow up but reach constant or vanishing values instead. So we impose

$$
Y_{1}^{I R}=0, \quad Y_{2}^{I R}=0, \quad Y_{4}^{U V}=0 .
$$

As a result of (5.9) and (5.8), the mode $\tilde{\phi}_{1}$ is identically zero. This yields $Y_{2}^{I R}=Y_{2}^{U V}$, $Y_{3}^{I R}=Y_{3}^{U V}$.

Since BPS M2 branes do not change the geometry of the Stenzel space but only the warp factor (much like BPS D3 branes also only change the warp factor and not the

\footnotetext{
${ }^{6}$ This combination multiplies a four-form field strength with one leg along $\nu$, one along $\sigma^{i}$ and two legs along two of the $\tilde{\sigma}^{j}$ directions which shrink in the IR $\left(e^{2 \beta_{0}} \sim r^{2}\right)$
} 
transverse geometry [50]) we expect the first-order perturbation to $e^{z+2 \beta}$ to vanish both in the UV and in the IR, and thus

$$
2 Y_{3}+e^{-3 z_{0}(0)} Y_{4}^{I R}+\frac{3}{2} m^{4} e^{-6 z_{0}(0)} Y_{6}^{I R}=0, \quad Y_{5}^{U V}=Y_{6}^{U V} .
$$

The constant $Y_{4}^{I R}$ is in turn determined by $Y_{4}^{U V}$. Furthermore, the fields $\tilde{\phi}_{5}, \tilde{\phi}_{6}$ now obey the corresponding homogeneous equations and the solution is found by replacing $\tilde{\lambda}_{5,6}$ by $Y_{5,6}$.

The mode $\tilde{\phi}_{4}$ corresponds to the first-order perturbation of the warp factor. We allow an $1 / r^{2}$ IR divergence, which means that $Y_{6}^{I R}$ doesn't necessarily need to vanish. We will see in a moment that this mode is related to the number $\Delta \mathcal{Q}_{M 2}$ of added M2 branes. But first, we note that this does not give rise to a singularity that would be associated with $\tilde{\phi}_{5}-4 \tilde{\phi}_{6}$, the perturbation to the term in $F_{4}(2.13)$ with legs on $\nu \wedge \sigma_{i} \wedge \tilde{\sigma}_{j} \wedge \tilde{\sigma}_{k}$. Indeed, the conditions we have imposed render this term harmless and independent of $Y_{6}^{I R}$ : $\tilde{\phi}_{5}-4 \tilde{\phi}_{6}=2 Y_{6}-2 Y_{6}+\mathcal{O}(r)=\mathcal{O}(r)$.

Given that $Y_{4}^{I R}$ first shows up in the $\mathcal{O}\left(r^{0}\right)$ part of the IR expansion of $\tilde{\phi}_{4}$ there is no restriction on it. Moreover, $Y_{5}$ does not arise in any of the divergent or constant pieces in the $\tilde{\phi}^{i}$ IR expansions, but requiring no exponentially divergent terms in the UV imposes $Y_{5}=Y_{6}$, in agreement with (5.10).

As a result, the perturbation corresponding to adding $\Delta \mathcal{Q}_{M 2}$ M2 branes at the tip is obtained by just setting $Y_{5}=Y_{6} \sim-\Delta \mathcal{Q}_{M 2}$. This perturbation causes the warp factor to diverge in the infrared as $-\Delta \mathcal{Q}_{M 2} / r^{2}$ while all the other $\phi^{i}$ change by sub-leading terms apart from $\phi^{5}$ and $\phi^{6}$ which shift by some $N$ related to $\Delta \mathcal{Q}_{M 2}$ through (5.5).

The UV expansion of the new warp factor is

$$
\begin{aligned}
H & =e^{3 z_{0}}\left(1+3 \tilde{\phi}_{4}\right) \\
& =\frac{16}{3} 2^{3 / 4} m^{2} e^{-9 r / 2}\left(1-6 Y_{3}\right)+\mathcal{O}\left(e^{-13 r / 2}\right) \\
& =\frac{16}{3} 2^{3 / 4} m^{2} e^{-9 r / 2}\left(1+3 e^{-3 z_{0}(0)} Y_{4}^{I R}+\frac{9}{2} m^{4} e^{-6 z_{0}(0)} Y_{6}\right)+\mathcal{O}\left(e^{-13 r / 2}\right),
\end{aligned}
$$

where in the last line we used (5.10), and one can see that $Y_{6}$ multiplies a $1 / \rho^{6}$ term, as expected from the exact solution.

\section{Constructing the anti-M2 brane solution}

In order to construct a first-order backreacted solution sourced by anti-M2 branes at the tip of the CGLP solution, the first necessary condition is that the force a probe M2 brane feels be nonzero, which implies:

$$
X_{4} \neq 0
$$

Furthermore, since the infrared is that of a smooth solution perturbed with smeared antiM2 branes, we require that no other field except those sourced by these anti-M2 branes have a divergent energy density in the infrared. 
Requiring no $\frac{1}{r^{2}}$ or stronger divergences in $\tilde{\phi}_{1}, \tilde{\phi}_{2}, \tilde{\phi}_{3}$ and $\tilde{\phi}_{6}$ immediately implies:

$$
\begin{aligned}
& X_{5}=-\frac{\sqrt{3}}{2} X_{4}, \\
& Y_{2}^{I R}=0, \\
& X_{1}=-2 X_{4},
\end{aligned}
$$

Barring any $\frac{1}{r}$ divergence in $\tilde{\phi}_{1,2}$ results in

$$
Y_{1}^{I R}=0 .
$$

The divergence in $\tilde{\phi}_{4}$ is now

$$
\tilde{\phi}_{4}=3^{1 / 4} \frac{\sqrt{3} Z_{0} m^{2} Y_{6}^{I R}-X_{4}}{r^{2}}+\mathcal{O}\left(r^{0}\right)
$$

and this is the proper divergence for the warp factor of anti-M2 branes spread on the $S^{4}$ in the infrared. The energy density that one can associate with this physical divergence is

$$
\rho(E) \sim \frac{d \tilde{\phi}_{4}}{d r} \sim \frac{1}{r^{6}}
$$

Another more subtle divergence in the infrared comes from the M-theory four-form field strength, which is

$$
G_{4}=d K(\tau) \wedge d x^{0} \wedge d x^{1} \wedge d x^{2}+m F_{4}
$$

where $(2.13)$

$$
\begin{aligned}
F_{4}= & \dot{f} d \tau \wedge \tilde{\sigma}_{1} \wedge \tilde{\sigma}_{2} \wedge \tilde{\sigma}_{3}+\dot{h} \epsilon^{i j k} d \tau \wedge \sigma_{i} \wedge \sigma_{j} \wedge \tilde{\sigma}_{k} \\
& +\frac{1}{2}(4 h-f) \epsilon^{i j k} \nu \wedge \sigma_{i} \wedge \tilde{\sigma}_{j} \wedge \tilde{\sigma}_{k}-6 h \nu \wedge \sigma_{1} \wedge \sigma_{2} \wedge \sigma_{3} .
\end{aligned}
$$

The unperturbed metric in the IR is regular and is given by

$$
d s^{2}=Z_{0}^{2 / 3} d s_{4}^{2}+\frac{1}{3^{3 / 4}} Z_{0}^{-1 / 3}\left[d r^{2}+\nu^{2}+\sigma_{i}^{2}+r^{2} \tilde{\sigma}_{i}^{2}\right],
$$

with the constant $Z_{0}$ given in (3.7). The vanishing metric components $g_{\tilde{\sigma} \tilde{\sigma}}$ lead to a divergent energy density from the four-form field strength components:

$$
\begin{aligned}
& F_{\nu \sigma \tilde{\sigma} \tilde{\sigma}} F_{\nu \sigma \tilde{\sigma} \tilde{\sigma}} g^{\nu \nu} g^{\sigma \sigma} g^{\tilde{\sigma} \tilde{\sigma}} g^{\tilde{\sigma} \tilde{\sigma}}=\frac{9 \sqrt{3} Z_{0}^{4 / 3} X_{4}^{2}}{r^{4}}+\mathcal{O}\left(r^{-2}\right) \\
& F_{r \tilde{\sigma} \tilde{\sigma} \tilde{\sigma}} F_{r \tilde{\sigma} \tilde{\sigma} \tilde{\sigma}} g^{r r} g^{\tilde{\sigma} \tilde{\sigma}} g^{\tilde{\sigma} \tilde{\sigma}} g^{\tilde{\sigma} \tilde{\sigma}}=\frac{81 \sqrt{3} Z_{0}^{3 / 4} X_{4}^{2}}{r^{4}}+\mathcal{O}\left(r^{-2}\right) \text {. }
\end{aligned}
$$

Unlike the analogous computations in IIB [22], when integrating these energy densities the factor of $\sqrt{-G} \sim r^{-3}$ is not strong enough to render the action finite. Hence, this singularity has both a divergent energy density, and a divergent action.

As discussed in the Introduction, if this singularity is physical then the perturbative solution we find corresponds to the first-order backreaction of a set of anti-M2 branes in the Stenzel-CGLP background. If this singularity is not physical, then our analysis indicates that anti-M2 branes cannot be treated as a perturbation of this background, and hints towards the fact that antibranes in backgrounds with positive brane charge dissolved in fluxes do not give rise to metastable vacua. 


\section{Acknowledgments}

We would like to thank Mariana Graña and Chris Herzog for interesting discussions. This work is supported in part by a Contrat de Formation par la Recherche of CEA/Saclay, the DSM CEA/Saclay, the grants ANR-07-CEXC-006 and ANR-08-JCJC-0001-0, and by the ERC Starting Independent Researcher Grant 240210 — String-QCD-BH.

\section{A Subtleties in section 2}

To justify our choice of integration constant in (2.20), we derive the expression for the non-dynamical scalar $K_{0}^{\prime}$ in two different ways. First of all, we use the expression (2.20) for $K^{\prime}$ that arises from its algebraic equation of motion. Inserting the zeroth-order expressions (2.27) of the fields appearing in this expression, we find

$$
K_{0}^{\prime}=-3 m^{2} \frac{\sinh (r)}{\cosh (r)^{4}} \frac{e^{-6 z_{0}(r)}}{(2+\cosh (2 r))^{3 / 4}} .
$$

On the other hand, let us proceed to see if this agrees with the expression obtained from the condition that the zeroth-order CGLP solution $K^{\prime}$ has to satisfy

$$
K_{0}^{\prime}=e^{-6 z_{0}(r)} \frac{d H_{0}}{d r}
$$

with $H_{0}$ solving

$$
\nabla_{8}^{2} H_{0}=-\frac{1}{2} m^{2}\left|F_{4}\right|^{2}
$$

This reduces to

$$
\frac{d H_{0}}{d r}=32^{3} m^{2} \frac{e^{2 \gamma_{0}}}{\sinh (2 r)^{3}}\left(\ell-\tanh (r)^{4}\right)
$$

and one must set $\ell=0$ in order for the solution to be regular. As a result,

$$
K_{0}^{\prime}=-3 m^{2} \frac{\sinh (r)}{\cosh (r)^{4}} \frac{e^{-6 z_{0}(r)}}{(2+\cosh (2 r))^{3 / 4}},
$$

in agreement with the expression for $K_{0}^{\prime}$ found above from the equation of motion for this non-dynamical field determined in term of $f_{0}$ and $h_{0}(2.27)$.

\section{B Behavior of $\tilde{\xi}$}

We collect here the infrared and ultraviolet asymptotic expansions of the exact solutions for $\tilde{\xi}^{i}$ which we have derived in section 4.1. 


\section{B.1 IR behavior of $\tilde{\xi}$}

The IR behavior of the $\tilde{\xi}_{a}$ 's is the following:

$$
\begin{aligned}
& \tilde{\xi}_{1}^{I R}= X_{1}+2 X_{4}\left[1-\frac{3^{1 / 4}}{2} m^{2} e^{-3 z_{0}(0)} r^{2}\right]+\mathcal{O}\left(r^{4}\right) \\
& \tilde{\xi}_{2}^{I R}= {\left[\frac{3}{2} X_{1}-\frac{4}{3 \sqrt{3}} X_{5}+\frac{7}{3} X_{4}\right]+\left[\frac{3}{2} X_{1}+\frac{8}{3 \sqrt{3}} X_{5}\right.} \\
&\left.+\frac{1}{3} X_{4}\left(13-103^{1 / 4} e^{-3 z_{0}(0)} m^{2}\right)\right] r^{2}+\mathcal{O}\left(r^{4}\right), \\
& \tilde{\xi}_{3}^{I R}=3^{1 / 4} e^{-3 z_{0}(0)} m^{2} X_{4} r^{2}+\mathcal{O}\left(r^{4}\right), \\
& \tilde{\xi}_{4}^{I R}=X_{4}\left[1-\frac{3^{1 / 4}}{2} m^{2} e^{-3 z_{0}(0)} r^{2}\right] \\
& \tilde{\xi}_{5}^{I R}=\frac{1}{r^{2}}\left[X_{5}+X_{4}\left(\frac{\sqrt{3}}{2}-\frac{3^{3 / 4}}{2} e^{-3 z_{0}(0)} m^{2}\right)\right] \\
&+ {\left[\frac{1}{6}\left(7 X_{5}+12 X_{6}\right)+X_{4}\left[\frac{17}{20 \sqrt{3}}-\frac{97}{12} 3^{3 / 4} e^{-3 z_{0}(0)} m^{2}\right.\right.} \\
&\left.\left.-\sqrt{6} e^{-3 z_{0}(0)} m^{2} \Pi\left(-\sqrt{3} ;-\arcsin \left(\frac{1}{3^{1 / 4}}\right) \mid-1\right)\right]-3^{3 / 4} e^{-3 z_{0}(0)} m^{2} X_{4} \log (r)\right] \\
&+\left[\frac{53}{120} X_{5}+\frac{1}{48} X_{4}\left(\frac{53}{5} \sqrt{3}+\frac{47}{5} 3^{3 / 4} e^{-3 z_{0}(0)} m^{2}\right)\right] r^{2}+\mathcal{O}\left(r^{4}\right), \\
& \tilde{\xi}_{6}^{I R}= \frac{2}{r^{2}}\left[2 X_{5}+\sqrt{3} X_{4}\right]+\left[\frac{4}{3} X_{5}+X_{4}\left(\frac{2}{\sqrt{3}}+3^{3 / 4} e^{-3 z_{0}(0)} m^{2}\right)\right] \\
&+ {\left[\frac{37}{30} X_{5}+X_{4}\left(\frac{37}{20 \sqrt{3}}-23^{3 / 4} e^{-3 z_{0}(0)} m^{2}\right)\right] r^{2}+\mathcal{O}\left(r^{4}\right) . }
\end{aligned}
$$

\section{B.2 UV behavior of $\tilde{\xi}$}

The UV behavior of the $\tilde{\xi}_{a}$ 's is as follows:

$$
\begin{aligned}
\tilde{\xi}_{1}^{U V}= & X_{1}+\frac{32}{3} 2^{3 / 4} m^{2} X_{4} e^{-3 z_{0}(0)} e^{-\frac{9}{2} r}+\mathcal{O}\left(e^{-13 r / 2}\right), \\
\tilde{\xi}_{2}^{U V}= & -\frac{3}{32} X_{3} e^{6 r}+\frac{3}{16} X_{3} e^{4 r}+\left[\frac{3}{8} X_{1}+\frac{3}{32} X_{3}+\frac{2}{3 \sqrt{3}}\left(X_{5}+X_{6}\right)\right] e^{2 r} \\
& +\left[\frac{3}{4} X_{1}-\frac{3}{8} X_{3}-\frac{8}{3 \sqrt{3}}\left(X_{5}+X_{6}\right)\right] \\
& +\left[\frac{3}{8} X_{1}+\frac{3}{32} X_{3}+\frac{2}{3 \sqrt{3}}\left(X_{5}+X_{6}\right)\right] e^{-2 r} \\
& +\left[\frac{3}{16} X_{3}+\frac{64}{3 \sqrt{3}} X_{6}\right] e^{-4 r}+\frac{32}{7} 2^{3 / 4} e^{-3 z_{0}(0)} m^{2} X_{4} e^{-9 r / 2} \\
& -\left[\frac{3}{32} X_{3}+\frac{256}{3 \sqrt{3}} X_{6}\right] e^{-6 r}+\mathcal{O}\left(e^{-13 r / 2}\right), \\
\tilde{\xi}_{3}^{U V}= & \frac{1}{8} X_{3} e^{6 r}-\frac{9}{8} X_{3} e^{2 r}+2 X_{3}-\frac{9}{8} X_{3} e^{-2 r} \\
& +\frac{32}{7} 2^{3 / 4} e^{-3 z_{0}(0)} m^{2} X_{4} e^{-9 r / 2}+\frac{1}{8} X_{3} e^{-6 r}+\mathcal{O}\left(e^{-13 r / 2}\right),
\end{aligned}
$$




$$
\begin{aligned}
\tilde{\xi}_{4}^{U V}= & \frac{16}{3} 2^{3 / 4} m^{2} X_{4} e^{-3 z_{0}(0)} e^{-\frac{9}{2} r}+\mathcal{O}\left(e^{-13 r / 2}\right), \\
\tilde{\xi}_{5}^{U V}= & \frac{1}{2}\left(X_{5}+X_{6}\right) e^{r}+\frac{5}{2}\left(X_{5}+X_{6}\right) e^{-r}+2\left(3 X_{5}-X_{6}\right) e^{-3 r} \\
& +2\left(5 X_{5}+X_{6}\right) e^{-5 r}-\frac{96}{13} 2^{3 / 4} \sqrt{3} e^{-3 z_{0}(0)} m^{2} X_{4} e^{-11 r / 2}+\mathcal{O}\left(e^{-13 r / 2}\right), \\
\tilde{\xi}_{6}^{U V}= & \left(X_{5}+X_{6}\right) e^{r}-7\left(X_{5}+X_{6}\right) e^{-r}-24\left(X_{5}-X_{6}\right) e^{-3 r} \\
& -8\left(5 X_{5}+7 X_{6}\right) e^{-5 r}-\frac{192}{13} 2^{3 / 4} \sqrt{3} m^{2} X_{4} e^{-3 z_{0}(0)} e^{-11 r / 2}+\mathcal{O}\left(e^{-13 r / 2}\right) .
\end{aligned}
$$

Open Access. This article is distributed under the terms of the Creative Commons Attribution Noncommercial License which permits any noncommercial use, distribution, and reproduction in any medium, provided the original author(s) and source are credited.

\section{References}

[1] K.A. Intriligator, N. Seiberg and D. Shih, Dynamical SUSY breaking in meta-stable vacua, JHEP 04 (2006) 021 [hep-th/0602239] [SPIRES].

[2] Y. Shadmi and Y. Shirman, Dynamical supersymmetry breaking, Rev. Mod. Phys. 72 (2000) 25 [hep-th/9907225] [SPIRES].

[3] A.E. Nelson and N. Seiberg, $R$ symmetry breaking versus supersymmetry breaking, Nucl. Phys. B 416 (1994) 46 [hep-ph/9309299] [SPIRES].

[4] K.A. Intriligator, N. Seiberg and D. Shih, Supersymmetry breaking, R-symmetry breaking and metastable vacua, JHEP 07 (2007) 017 [hep-th/0703281] [SPIRES].

[5] K.A. Intriligator and N. Seiberg, Lectures on supersymmetry breaking, Class. Quant. Grav. 24 (2007) S741 [hep-ph/0702069] [SPIRES].

[6] I. Bena, E. Gorbatov, S. Hellerman, N. Seiberg and D. Shih, A note on (meta)stable brane configurations in MQCD, JHEP 11 (2006) 088 [hep-th/0608157] [SPIRES].

[7] H. Ooguri and Y. Ookouchi, Meta-stable supersymmetry breaking vacua on intersecting branes, Phys. Lett. B 641 (2006) 323 [hep-th/0607183] [SPIRES].

[8] S. Franco, I. Garcia-Etxebarria and A.M. Uranga, Non-supersymmetric meta-stable vacua from brane configurations, JHEP 01 (2007) 085 [hep-th/0607218] [SPIRES].

[9] E. Witten, Solutions of four-dimensional field theories via M-theory, Nucl. Phys. B 500 (1997) 3 [hep-th/9703166] [SPIRES].

[10] K. Hori, H. Ooguri and Y. Oz, Strong coupling dynamics of four-dimensional $N=1$ gauge theories from M-theory fivebrane, Adv. Theor. Math. Phys. 1 (1998) 1 [hep-th/9706082] [SPIRES].

[11] A. Brandhuber, N. Itzhaki, V. Kaplunovsky, J. Sonnenschein and S. Yankielowicz, Comments on the M-theory approach to $N=1 S Q C D$ and brane dynamics, Phys. Lett. B 410 (1997) 27 [hep-th/9706127] [SPIRES].

[12] A. Giveon and D. Kutasov, Brane dynamics and gauge theory, Rev. Mod. Phys. 71 (1999) 983 [hep-th/9802067] [SPIRES].

[13] S. Kachru, J. Pearson and H.L. Verlinde, Brane/flux annihilation and the string dual of a non-supersymmetric field theory, JHEP 06 (2002) 021 [hep-th/0112197] [SPIRES]. 
[14] O. DeWolfe, S. Kachru and H.L. Verlinde, The giant inflaton, JHEP 05 (2004) 017 [hep-th/0403123] [SPIRES].

[15] I.R. Klebanov and M.J. Strassler, Supergravity and a confining gauge theory: duality cascades and $\chi_{S B}$-resolution of naked singularities, JHEP 08 (2000) 052 [hep-th/0007191] [SPIRES].

[16] R.C. Myers, Dielectric-branes, JHEP 12 (1999) 022 [hep-th/9910053] [SPIRES].

[17] J. Polchinski and M.J. Strassler, The string dual of a confining four-dimensional gauge theory, hep-th/0003136 [SPIRES].

[18] I.R. Klebanov and S.S. Pufu, M-branes and metastable states, arXiv: 1006. 3587 [SPIRES].

[19] M. Stenzel, Ricci-flat metrics on the complexification of a compact rank one symmetric space, Manuscripta Math. 80 (1993) 1.

[20] M. Cvetič, G.W. Gibbons, H. Lü and C.N. Pope, Ricci-flat metrics, harmonic forms and brane resolutions, Commun. Math. Phys. 232 (2003) 457 [hep-th/0012011] [SPIRES].

[21] I. Bena, The M-theory dual of a 3 dimensional theory with reduced supersymmetry, Phys. Rev. D 62 (2000) 126006 [hep-th/0004142] [SPIRES].

[22] I. Bena, M. Graña and N. Halmagyi, On the existence of meta-stable vacua in Klebanov-Strassler, JHEP 09 (2010) 087 [arXiv:0912.3519] [SPIRES].

[23] S. Kachru et al., Towards inflation in string theory, JCAP 10 (2003) 013 [hep-th/0308055] [SPIRES].

[24] O. DeWolfe, S. Kachru and M. Mulligan, A gravity dual of metastable dynamical supersymmetry breaking, Phys. Rev. D 77 (2008) 065011 [arXiv:0801.1520] [SPIRES].

[25] I.R. Klebanov and A.A. Tseytlin, Gravity duals of supersymmetric $\mathrm{SU}(N) \times \mathrm{SU}(N+M)$ gauge theories, Nucl. Phys. B 578 (2000) 123 [hep-th/0002159] [SPIRES].

[26] P. McGuirk, G. Shiu and Y. Sumitomo, Non-supersymmetric infrared perturbations to the warped deformed conifold, Nucl. Phys. B 842 (2010) 383 [arXiv:0910.4581] [SPIRES].

[27] G.T. Horowitz and R.C. Myers, The value of singularities, Gen. Rel. Grav. 27 (1995) 915 [gr-qc/9503062] [SPIRES].

[28] V. Borokhov and S.S. Gubser, Non-supersymmetric deformations of the dual of a confining gauge theory, JHEP 05 (2003) 034 [hep-th/0206098] [SPIRES].

[29] G. Papadopoulos and A.A. Tseytlin, Complex geometry of conifolds and 5-brane wrapped on 2-sphere, Class. Quant. Grav. 18 (2001) 1333 [hep-th/0012034] [SPIRES].

[30] D. Cassani and A.F. Faedo, A supersymmetric consistent truncation for conifold solutions, Nucl. Phys. B 843 (2011) 455 [arXiv: 1008.0883] [SPIRES].

[31] I. Bena, G. Giecold, M. Graña, N. Halmagyi and F. Orsi, Supersymmetric consistent truncations of IIB on T(1,1), JHEP 04 (2011) 021 [arXiv: 1008.0983] [SPIRES].

[32] J.M. Maldacena and H.S. Nastase, The supergravity dual of a theory with dynamical supersymmetry breaking, JHEP 09 (2001) 024 [hep-th/0105049] [SPIRES].

[33] P. McFadden and K. Skenderis, The holographic universe, J. Phys. Conf. Ser. 222 (2010) 012007 [arXiv: 1001.2007] [SPIRES].

[34] C. Krishnan and S. Kuperstein, The mesonic branch of the deformed conifold, JHEP 05 (2008) 072 [arXiv:0802.3674] [SPIRES]. 
[35] S.S. Pufu, I.R. Klebanov, T. Klose and J. Lin, Green's functions and non-singlet glueballs on deformed conifolds, J. Phys. A 44 (2011) 055404 [arXiv: 1009.2763] [SPIRES].

[36] J. Blaback et al., Smeared versus localised sources in flux compactifications, JHEP 12 (2010) 043 [arXiv: 1009.1877] [SPIRES].

[37] A. Giveon, D. Kutasov and O. Lunin, Spontaneous SUSY breaking in various dimensions, Nucl. Phys. B 822 (2009) 127 [arXiv:0904.2175] [SPIRES].

[38] G. Giecold, unpublished.

[39] M. Cvetič, G.W. Gibbons, H. Lü and C.N. Pope, Supersymmetric non-singular fractional D2-branes and NS-NS 2-branes, Nucl. Phys. B 606 (2001) 18 [hep-th/0101096] [SPIRES].

[40] G.C. Giecold, Finite-temperature fractional D2-branes and the deconfinement transition in $2+1$ dimensions, JHEP 03 (2010) 109 [arXiv:0912.1558] [SPIRES].

[41] S. Kuperstein and J. Sonnenschein, Analytic non-supersymmetric background dual of a confining gauge theory and the corresponding plane wave theory of hadrons, JHEP 02 (2004) 015 [hep-th/0309011] [SPIRES].

[42] A. Ceresole, G. Dall'Agata, R. D'Auria and S. Ferrara, M-theory on the Stiefel manifold and $3 D$ conformal field theories, JHEP 03 (2000) 011 [hep-th/9912107] [SPIRES].

[43] P. Candelas and X.C. de la Ossa, Comments on conifolds, Nucl. Phys. B 342 (1990) 246 [SPIRES].

[44] D. Martelli and J. Sparks, $A d S_{4} / C F T_{3}$ duals from M2-branes at hypersurface singularities and their deformations, JHEP 12 (2009) 017 [arXiv:0909.2036] [SPIRES].

[45] D.L. Jafferis, Quantum corrections to $N=2$ Chern-Simons theories with flavor and their $A d S_{4}$ duals, arXiv:0911.4324 [SPIRES].

[46] I. Bena, G. Giecold, M. Graña and N. Halmagyi, On the inflaton potential from antibranes in warped throats, arXiv: 1011.2626 [SPIRES].

[47] T. Banks, M.R. Douglas, G.T. Horowitz and E.J. Martinec, AdS dynamics from conformal field theory, hep-th/9808016 [SPIRES].

[48] V. Balasubramanian, P. Kraus, A.E. Lawrence and S.P. Trivedi, Holographic probes of Anti-de Sitter space-times, Phys. Rev. D 59 (1999) 104021 [hep-th/9808017] [SPIRES].

[49] A. Bergman and C.P. Herzog, The volume of some non-spherical horizons and the AdS/CFT correspondence, JHEP 01 (2002) 030 [hep-th/0108020] [SPIRES].

[50] M. Graña and J. Polchinski, Supersymmetric three-form flux perturbations on AdS $S_{5}$, Phys. Rev. D 63 (2001) 026001 [hep-th/0009211] [SPIRES]. 